\title{
Atendimento pré-hospitalar: caracterização das ocorrências de acidente de trânsito*
}

\author{
Pre-hospital Care: Characteristics of Traffic Accidents \\ Atención prehospitalaria: caracterización de las ocurrencias de accidentes de tránsito
}

Waleska Antunes da Porciúncula Pereira ${ }^{1}$, Maria Alice Dias da Silva Lima ${ }^{2}$

\begin{abstract}
RESUMO
Objetivo: identificar as ocorrências atendidas por um serviço de atendimento pré-hospitalar e caracterizar as decorrentes de corte acidente de trânsito em relação ao horário, dia da semana e configuração da equipe envolvida no atendimento. Métodos: estudo descritivo transversal, com análise de 6.430 ocorrências de solicitação de socorro atendidas de julho a setembro de 2003. Resultados: a incidência de trauma foi $35,2 \%$ sendo $57,9 \%$ decorrentes de acidentes de trânsito. A maioria das ocorrências aconteceu à tarde, em todos os dias da semana. A equipe de suporte básico, constituída por auxiliar ou técnico de enfermagem e motorista, foi a que mais realizou atendimentos (84,5\%). A enfermeira participou em $11,2 \%$ das ocorrências, sendo $4,3 \%$ na equipe de suporte avançado e o médico em $8,3 \%$. Conclusão: os resultados destacam o envolvimento da equipe de suporte básico no atendimento pré-hospitalar e indicam a necessidade de prevenção desses agravos e de qualificação dos trabalhadores para estruturação do trabalho baseado na interdisciplinaridade.
\end{abstract}

Descritores: Acidentes de trânsito; Serviços médicos de emergência; Socorro de urgência

\begin{abstract}
Objectives: to identify types of occurrence registered by a pre-hospital care unit and to characterize occurrences from traffic accidents. Methods: a descriptive cross-sectional study was used to analyze 6,430 pre-hospital calls from July to September, 2003. Results: the occurrences were classified as trauma $(35.2 \%$, ) or traffic accidents $(57.9 \%)$. Most occurrences took place in the afternoon and were common in all days of the week. The basic pre-hospital care team, consisting of a licensed practical nurse or associate degree nurse and an ambulance driver, was the team that answered most emergency calls (84.5\%). A professional nurse participated in only $11.2 \%$ of the occurrences, and great part of these occurrences (4.27\%) was answered by the advanced pre-hospital care team. A physician participated in only $8.3 \%$ of occurrences. Conclusion: the basic pre-hospital care team was involved in the majority of emergency calls. This suggests a need of new strategies for preventing victim's complications and better ways to qualify pre-hospital care team members for a quality interdisciplinarybased work.
\end{abstract}

Keywords: Traffic accidents; Emergency medical services; Emergency relief

\section{RESUMEN}

Objetivo: identificar las ocurrencias atendidas en un servicio de atención prehospitalaria y caracterizar las consecuentes de accidente de tránsito en relación al horario, día de la semana y configuración del equipo involucrado en la atención. Métodos: estudio descriptivo de corte transversal, con análisis de 6,430 ocurrencias de solicitud de socorro atendidas de julio a septiembre del 2003. Resultados: la incidencia de trauma fue del $35.2 \%$ de los cuales $57.9 \%$ a causa de accidentes de tránsito. La mayoría de las ocurrencias suceden en la tarde de todos los días de la semana. El equipo de soporte básico, constituido por el auxiliar o técnico de enfermería y el chofer, es el que más atenciones realiza (84.5\%). La enfermera participa en el 11.2\% de las ocurrencias, siendo un 4.27\% en el equipo de soporte avanzado y el médico en el $8.32 \%$. Conclusión: Los resultados destacan el involucramiento del equipo de soporte básico en la atención prehospitalaria e indican la necesidad de prevención de esos daños y de calificación de los trabajadores para la estructuración del trabajo basado en la interdisciplinaridad.

Descriptores: Accidentes de tránsito; Servicios médicos de emergencia; urgências médicas

\footnotetext{
${ }^{*}$ Estudo empírico de base extraído da Dissertação de Mestrado defendida na Escola de Enfermagem da Universidade Federal do Rio Grande do Sul - UFRGS - Porto Alegre (RS), Brasil.

${ }^{1}$ Mestre em Enfermagem pela Universidade Federal do Rio Grande do Sul. Docente da Escola de Saúde Pública do Rio Grande do Sul - UFRGS - Porto Alegre (RS), Brasil.

${ }^{2}$ Doutora em Enfermagem. Professora Adjunto, Escola de Enfermagem, Universidade Federal do Rio Grande do Sul - UFRGS - Porto Alegre (RS), Brasil.
} 


\section{INTRODUÇÃO}

Os acidentes de trânsito têm sido alvo de grande preocupação no Brasil e no mundo, pelo elevado número de vítimas jovens que atingem e pelos impactos sociais, econômicos e pessoais que provocam. No Rio Grande do Sul, a mortalidade por causas externas foi a mais acentuada na faixa etária de 1 a 39 anos, em 2002. Esses óbitos foram decorrentes de homicídios e de acidentes de transporte, numa tendência linear ascendente, e são responsáveis pelos maiores índices do indicador anos potenciais de vida perdidos, visto que atingiram uma população extremamente jovem ${ }^{(1)}$.

No Brasil, as causas externas representaram a terceira causa de morte no país, no ano de 2000 , com $14,5 \%$ do total de mortes. Nas duas últimas décadas, os acidentes de trânsito foram a principal razão das mortes por causas externas no Rio Grande do Sul, enquanto nas outras regiões do Brasil, foram superados pelos homicídios. Os acidentes de trânsito, em 2000, representaram 29,4\% dos óbitos por causas externas, no Estado, enquanto os homicídios chegaram a $25,4 \%{ }^{(2)}$.

$\mathrm{O}$ serviço de atendimento pré-hospitalar (APH) envolve todas as ações que ocorrem antes da chegada do paciente ao ambiente hospitalar, e pode influir positivamente nas taxas de morbidade e mortalidade por trauma. A assistência qualificada na cena do acidente, o transporte e a chegada precoce ao hospital são fundamentais para que a vítima chegue ao hospital com vida ${ }^{(3)}$. O APH é realizado através de duas modalidades: o suporte básico à vida, que se caracteriza por não realizar manobras invasivas e o suporte avançado à vida, que possibilita procedimentos invasivos de suporte ventilatório e circulatório ${ }^{(4)}$.

Esse cenário que envolve o APH tem sido objeto de alguns estudos epidemiológicos sobre a população atendida, incluindo o perfil das vítimas atendidas, diagnóstico de lesões, coeficientes de letalidade, entre outros aspectos. É necessário ampliar o conhecimento em relação às ocorrências de acidente de trânsito e ao papel que o atendimento pré-hospitalar vem desenvolvendo.

Nesse sentido, os objetivos deste estudo foram identificar as ocorrências atendidas por um serviço de atendimento pré-hospitalar,em um grande centro urbano do sul do País; e caracterizar as ocorrências decorrentes de acidente de trânsito, em relação ao horário em que aconteceram, dia da semana e configuração da equipe envolvida no atendimento.

\section{MÉTODOS}

Neste estudo descritivo de corte transversal utilizamos, como fonte de informações para coleta de dados, as fichas de regulação, que foram preenchidas pelos auxiliares de regulação, rádio operadores e médicos reguladores, em um serviço de atendimento pré-hospitalar de Porto Alegre, no período de julho a setembro de 2003. A ficha de regulação é gerada para cada chamado recebido, sejam as solicitações de socorro e de transporte que são atendidas, ou as que apenas recebem orientação do médico, por telefone, ou mesmo as que não são atendidas por falta de recursos no momento, ou por não serem pertinentes. Nessa ficha verificamos os seguintes itens: motivo da solicitação de socorro, descrição da ocorrência, data, horário do chamado, equipe que foi enviada para o atendimento, categorias profissionais que compõem a equipe.

Foram verificadas 12.729 fichas de regulação, sendo que 5.247 não geraram ocorrência, 6.430 referiam-se a ocorrências de solicitação de socorro e 1.052 de transporte simples ou medicalizado de pacientes. Consideramos como unidade para caracterização da ocorrência, o acidente, que pode ter sido atendido por uma ou mais equipes, cada uma delas gerando uma ficha de regulação, mas sem se constituir, entretanto, em uma nova ocorrência.

As equipes de suporte básico e suporte avançado foram definidas a partir da configuração da equipe ou equipes envolvidas no atendimento, sem considerar as características do veículo utilizado, pois a ambulância de suporte básico, quando recebe o apoio do veículo rápido, equipara-se à de suporte avançado, da mesma forma que esta, quando tripulada por uma equipe composta por motorista, auxiliar ou técnico de enfermagem e mesmo a enfermeira, sem a presença do médico, atua como suporte básico.

O tratamento e análise dos dados foram realizados de forma descritiva, com a distribuição absoluta e relativa das informações obtidas nas fichas de regulação. Foram empregados na pesquisa percentuais e médias, sendo apresentados os resultados na forma tabular.

Obteve-se autorização institucional para exame das fichas de regulação e coleta de dados e aprovação do Comitê de Ética em Pesquisa do Hospital de Pronto Socorro Municipal de Porto Alegre.

\section{RESULTADOS}

Entre as 6.430 fichas referentes às ocorrências de solicitação de socorro atendidas, a incidência do trauma foi de $35,2 \%$ e a incidência de ocorrências não traumáticas caracterizadas como clínicas, obstétricas e psiquiátricas, foi de $64,8 \%$.

No que se refere aos acidentes de trânsito, verificamos que representaram 57,9\% das ocorrências de trauma, sendo superior às quedas $(26,9 \%)$, violência interpessoal que engloba a agressão e os ferimentos por arma branca e arma de fogo $(12,1 \%)$ e outras $(3,1 \%)$, conforme 
apresentamos na Tabela 1. Na categoria outras causas agrupamos ocorrências diversas, tais como queimadura, corte, incêndio, choque elétrico, soterramento, mordida de cão.

Tabela 1 - Ocorrências atendidas por um serviço de atendimento pré-hospitalar, segundo tipo de causa e mês de ocorrência. Porto Alegre, julho a setembro de 2003

\begin{tabular}{lrrrrr}
\hline \multirow{2}{*}{$\begin{array}{l}\text { MÊS } \\
\text { OCORRÊNCIA }\end{array}$} & \multicolumn{1}{c}{ JUL } & AGO & \multicolumn{2}{l}{ SET } & \multicolumn{2}{c}{ TOTAL } \\
\cline { 2 - 6 } & $\mathrm{n}$ & $\mathrm{n}$ & $\mathrm{n}$ & $\mathrm{n}$ & $\%$ \\
\hline Acidente de trânsito & 411 & 491 & 408 & 1310 & 57,9 \\
Queda & 182 & 222 & 206 & 610 & 26,9 \\
Violência interpessoal & 93 & 90 & 91 & 274 & 12,1 \\
Outras & 25 & 25 & 20 & 70 & 3,1 \\
\hline TOTAL & 711 & 828 & 725 & 2264 & 100,0 \\
\hline
\end{tabular}

Os acidentes de trânsito relacionaram-se aos atropelamentos, numa proporção de $30,7 \%$ das ocorrências e às colisões $69,3 \%$. Na categoria colisão foram englobadas: colisão, abalroamento, capotagem e choque com objeto fixo, envolvendo qualquer veículo (ônibus, carro, lotação, caminhão, carroça, motocicleta, bicicleta).

As ocorrências aconteceram com regulação médica e, conforme os dados da Tabela 2, apresentaram maior freqüência $(37,7 \%)$ no turno da tarde, seguido do turno da noite $(25,5 \%)$ e manhã $(23,8 \%)$. O turno da madrugada, compreendido entre 1 e 6 horas, teve a menor incidência de ocorrências $(13,0 \%)$.

Tabela 2 - Ocorrências atendidas por um serviço de atendimento pré-hospitalar, segundo turno e mês. Porto Alegre, julho a setembro de 2003

\begin{tabular}{lccccccccc}
\hline \multicolumn{1}{c}{\begin{tabular}{c} 
MÊS \\
\multicolumn{1}{c}{ TURNO }
\end{tabular}} & \multicolumn{1}{c}{$\mathrm{JUL}$} & \multicolumn{1}{c}{ AGO } & \multicolumn{2}{c}{ SET } & \multicolumn{2}{c}{ TOTAL } \\
\cline { 2 - 9 } & $\mathrm{n}$ & $\%$ & $\mathrm{n}$ & $\%$ & $\mathrm{n}$ & $\%$ & $\mathrm{n}$ & $\%$ \\
\hline $\begin{array}{l}\text { Madrugada } \\
\text { (1h às 6h 59m) }\end{array}$ & 58 & 14,1 & 57 & 11,6 & 56 & 13,7 & 171 & 13,0 \\
$\begin{array}{l}\text { Manhã } \\
\text { (7h às 12h 59m) }\end{array}$ & 94 & 22,9 & 120 & 24,5 & 98 & 24,0 & 312 & 23,8 \\
$\begin{array}{l}\text { Tarde } \\
(13 \mathrm{~h} \text { às 18h 59m) }\end{array}$ & 154 & 37,5 & 199 & 40,5 & 142 & 34,8 & 495 & 37,7 \\
$\begin{array}{l}\text { Noite } \\
(19 \mathrm{~h} \text { à 0h 59m) }\end{array}$ & 105 & 25,5 & 115 & 23,4 & 112 & 27,5 & 332 & 25,5 \\
TOTAL & 411 & 100,0 & 491100,0 & 408 & 100,0 & 1310 & 100,0 \\
\hline
\end{tabular}

Os atendimentos às ocorrências de acidente de trânsito distribuíram-se em todos os dias da semana, com relativa concentração nos finais de semana $(32,2 \%)$ no mês de agosto. No entanto, essa tendência não se repetiu nos outros meses analisados, visto que julho apresentou uma concentração maior das ocorrências na quinta-feira $(19,4 \%)$ e quarta-feira $(17,5 \%)$, totalizando $36,9 \%$ enquanto no mês de setembro ocorreram na segundafeira e sábado $(33,1 \%)$.
Tabela 3 - Ocorrências de acidente de trânsito atendidas por um serviço de atendimento pré-hospitalar, segundo dia da semana e mês de ocorrência. Porto Alegre, julho a setembro de 2003

\begin{tabular}{lrrrrrrrrr}
\hline \multirow{2}{*}{ MÊS } & \multicolumn{2}{c}{$\mathrm{JUL}$} & \multicolumn{2}{c}{ AGO } & \multicolumn{2}{c}{ SET } & \multicolumn{3}{c}{ TOTAL } \\
\cline { 2 - 10 } DIA SEMANA & $\mathrm{n}$ & \multicolumn{1}{c}{$\%$} & $\mathrm{n}$ & $\%$ & $\mathrm{n}$ & $\%$ & $\mathrm{n}$ & $\%$ \\
\hline Domingo & 57 & 13,9 & 82 & 16,7 & 49 & 12,0 & 188 & 14,3 \\
Segunda-feira & 36 & 8,7 & 63 & 12,8 & 68 & 16,7 & 167 & 12,8 \\
Terça-feira & 59 & 14,3 & 55 & 11,2 & 53 & 13,0 & 167 & 12,8 \\
Quarta-feira & 72 & 17,5 & 65 & 12,3 & 57 & 13,9 & 194 & 14,8 \\
Quinta-feira & 80 & 19,5 & 63 & 12,8 & 56 & 13,7 & 199 & 15,2 \\
Sexta-feira & 43 & 10,5 & 87 & 17,7 & 58 & 14,2 & 188 & 14,3 \\
Sábado & 64 & 15,6 & 76 & 15,5 & 67 & 16,4 & 207 & 15,8 \\
\hline TOTAL & 411100,0 & 491100,0 & 408 & 100,0 & 1310 & 100,0 \\
\hline
\end{tabular}

Em relação às equipes envolvidas no atendimento, identificou-se que a de suporte básico, constituída por um auxiliar ou técnico de enfermagem e um motorista, foi a que mais realizou os atendimentos (1.107) atingindo um percentual de $84,5 \%$. Em 11,2\% das ocorrências (147), a enfermeira participou da equipe, sendo que em $6,2 \%$ (82) estava em supervisão, em $0,7 \%$ (9) substituindo o auxiliar de enfermagem e em 4,3\% (56) compondo a equipe de suporte avançado, junto com o médico. A participação do médico aconteceu em 8,3\% das ocorrências (109).

Tabela 4 - Ocorrências de acidente de trânsito atendidas por um serviço de atendimento pré-hospitalar, segundo configuração da equipe e mês de ocorrência. Porto Alegre, julho a setembro de 2003.

\begin{tabular}{|c|c|c|c|c|c|c|c|c|}
\hline \multirow{2}{*}{$\begin{array}{c}\text { MÊS } \\
\text { EQUIPE* }\end{array}$} & \multicolumn{2}{|c|}{ JUL } & \multicolumn{2}{|c|}{ AGO } & \multicolumn{2}{|c|}{ SET } & \multicolumn{2}{|c|}{ TOTAL } \\
\hline & $\mathrm{n}$ & $\%$ & $\mathrm{n}$ & $\%$ & $\mathrm{n}$ & $\%$ & $\mathrm{n}$ & $\%$ \\
\hline AMo & 338 & 82,2 & 438 & 89,2 & 331 & 81,1 & 1107 & 84,5 \\
\hline EAMo & 30 & 7,3 & 20 & 4,1 & 32 & 7,8 & 82 & 6,3 \\
\hline EMo & 4 & 1,0 & 1 & 0,2 & 4 & 1,0 & 9 & 0,7 \\
\hline IAMo & 2 & 0,5 & 1 & 0,2 & - & - & 3 & 0,2 \\
\hline MeAMo & 23 & 5,6 & 16 & 3,3 & 14 & 3,4 & 53 & 4,0 \\
\hline MeEAMo & 13 & 3,2 & 15 & 3,0 & 26 & 6,4 & 54 & 4,1 \\
\hline MeEIAMo & 1 & 0,2 & - & - & 1 & 0,3 & 2 & 0,2 \\
\hline TOTAL & 411 & 100, & 491 & 100,0 & 408 & 20 & 1510 & 00,0 \\
\hline
\end{tabular}

Legenda: As siglas utilizadas formaram-se a partir da sigla criada para cada uma das categorias profissionais envolvidas: A - auxiliar ou técnico de enfermagem; Mo - motorista; $\mathrm{E}$ - enfermeira; Me - médico; I - interno (acadêmico de Medicina).

\section{DISCUSSÃO}

Os serviços de APH no País e no Rio Grande do Sul surgiram a partir de demandas em situações de emergência relacionadas ao trauma. Verificamos que outras demandas reprimidas, não atendidas pela Rede Pública de Saúde, 
passaram a ser atendidas pelo pré-hospitalar, representando um acréscimo nos atendimentos classificados como intercorrências clínicas, que envolvem afecções neurológicas, problemas cardíacos e respiratórios, atendimentos obstétricos e psiquiátricos.

Estudos realizados nesse serviço de atendimento préhospitalar, nos períodos de novembro de 1995 a abril de $1996^{(5)}$ e de janeiro a junho de $1999^{(6)}$ revelaram uma incidência de ocorrências de trauma de 40\% em 1996 e $28,1 \%$ em 1999. Indicaram também a predominância de acidente de trânsito em relação ao conjunto das ocorrências de trauma, num percentual de $51 \%$ e $54 \%$, respectivamente. A incidência de $57,9 \%$ constatada neste estudo mostra um aumento dessas ocorrências, seguindo a tendência linear ascendente da mortalidade decorrente desse agravo, apontada pelos indicadores de mortalidade, nas estatísticas de saúde do Rio Grande do Sul ${ }^{(1)}$.

Analisando dados ${ }^{(7)}$ referentes aos acidentes de trânsito com vítimas, ocorridos em Porto Alegre, identificou-se uma diminuição nos anos de 1998 e 1999, possivelmente em função da aprovação do Código de Trânsito Brasileiro. A partir de 1999, houve um aumento considerável desses acidentes, passando de 4.891, em 1999, para 6.156, em 2003. Em relação aos meses de julho, agosto e setembro de 2003, foram registrados 549, 579 e 520 acidentes com vítimas numa média diária que chegou, respectivamente, a 18,7 em agosto, e em julho 17,7 e 17,3 em setembro. Comparando estes dados com o número de ocorrências atendidas pelo serviço de atendimento pré-hospitalar, constatamos que os atendimentos aconteceram em 74 a $81 \%$ dos acidentes registrados em Porto Alegre, enquanto o restante foi realizado pelo Corpo de Bombeiros, Brigada Militar, Empresa Pública de Transporte e Circulação, serviços privados, ou a vítima foi removido por meios próprios. Esses dados demonstram a influência do APH no atendimento às vítimas de acidentes de trânsito, podendo repercutir positivamente na redução da morbimortalidade decorrente desse agravo.

Apesar de as colisões serem mais freqüentes, observamos que os acidentes envolvendo pedestres tendem a ser mais graves. As estatísticas referentes aos acidentes de trânsito destacam o pedestre, vítima de atropelamento (cerca de $40 \%$ das mortes) como o elemento mais vulnerável no trânsito ${ }^{(2)}$. Estudos realizados em Brasília ${ }^{(8)}$ e em Curitiba ${ }^{(9)}$ apresentam dados semelhantes em relação à caracterização dos acidentes quanto ao tipo, assim como mostram que os atropelamentos, apesar de serem em menor quantidade, são os responsáveis pela maior quantidade de óbitos decorrentes de trauma.

Em Londrina, Paraná, no período de janeiro a junho de 1996, identificou-se que o horário de maior média de vítimas/dia variava conforme os dias da semana. Aos sábados e domingos o período com o maior número de vítimas foi das 20 às 21 horas, enquanto nos outros dias da semana o pico foi observado no horário das 18 às 19 horas, assim como no início do turno da manhã, das 6 às 9 horas, e no início da tarde, das 13 às 14 horas ${ }^{(10)}$. Acreditamos que a maior incidência de ocorrências atendidas no turno da tarde deva-se ao maior fluxo de veículos e pedestres nesse horário, o que aumenta as chances de ocorrerem acidentes de trânsito.

Os estudos já realizados não permitem conclusões quanto a maior incidência de atendimento a ocorrências de acidentes de trânsito em algum dos dias da semana, sendo necessária a realização de um estudo abrangendo um espaço de tempo maior, para avaliar não só o período em que ocorrem os acidentes, mas também suas características e gravidade. Os acidentes nas rodovias apresentam variações de acordo com o dia da semana, com maior incidência no final de semana, conforme dados de 1993 a $1994^{(11)}$. No entanto, esse aumento no fluxo de veículos nas rodovias, que ocorre nos finais de semana, contribuindo para o aumento na incidência de acidentes de trânsito, não se repete nos centros urbanos, pois sua dinâmica é diferente.

Em Porto Alegre, de janeiro a junho de 1999, as remoções de pacientes hospitalizados representaram $46,9 \%$ dos atendimentos, sendo $62,8 \%$ das remoções realizadas por equipe socorrista, constituída por motorista e auxiliar de enfermagem, enquanto $7,4 \%$ foram remoções medicalizadas ${ }^{(6)}$.

O perfil do atendimento, com ênfase no trabalho das equipes de suporte básico, cumpre a finalidade do préhospitalar que é de fundamental importância na prevenção das lesões secundárias, devendo ser realizado no menor tempo e de forma que mantenha a estabilidade clínica da vítima até a chegada ao hospital ${ }^{(11)}$.

Esse cenário encontra respaldo em estudos internacionais ${ }^{(12)}$, os quais sugerem que o atendimento de suporte básico de vida, no ambiente pré-hospitalar diminui a mortalidade dos pacientes vítimas de trauma, quando comparado com o suporte avançado. Os autores ressaltam que em situações de trauma o tempo é essencial e, nesses estudos, verificou-se que quanto mais tempo é gasto na cena, realizando procedimentos avançados, mais tardiamente o paciente recebe o atendimento definitivo, tendo, conseqüentemente, os piores resultados.

A participação da enfermeira no atendimento préhospitalar é recente e, constitui-se numa prática nova para os padrões de Enfermagem tradicional. A enfermeira passou a atuar na assistência direta às vítimas atendidas no pré-hospitalar a partir da década de 90 , quando foram incorporadas aos serviços das unidades de suporte avançado $^{(13)}$. No atendimento às vítimas de acidente de trânsito identificou-se uma dimensão ainda não relatada, caracterizada como supervisão, em que a enfermeira se 
soma à equipe no atendimento de suporte básico, participando do atendimento em si e colhendo subsídios para aprimorar esse trabalho, tanto no campo técnico como no das relações.

\section{CONCLUSÃO}

Os dados epidemiológicos e de caracterização das ocorrências que foram analisados demonstram a relevância do atendimento às vítimas de acidentes de trânsito.

Constatou-se que o atendimento pré-hospitalar em todas as suas ações, seja de remoção ou de atendimento de urgência, é realizado, majoritariamente, pelas equipes de suporte básico. A enfermeira ampliou o seu espaço de atuação nesse campo, nos últimos anos. Além do trabalho de gerência e administração, ela tem maior inserção no trabalho assistencial, seja no âmbito do atendimento com suporte avançado ou suporte básico.

Em algumas situações, o atendimento pré-hospitalar torna-se a "porta de entrada" no Sistema de Saúde. Nesse sentido, uma análise mais detalhada dos atendimentos realizados no serviço de atendimento pré-hospitalar poderia mostrar o funcionamento do sistema de saúde no Município, apontando as situações em que se torna necessária alguma intervenção específica.

Identifica-se a necessidade de aprofundarmos o conhecimento nessa área, buscando subsídios para a prevenção desses agravos, a formação e qualificação dos trabalhadores e a estruturação do trabalho baseado na interdisciplinariedade.

\section{REFERÊNCIAS}

1. Rio Grande do Sul. Secretaria da Saúde. Estatísticas de saúde: mortalidade 2001. Porto Alegre: Coordenadoria de
Informações em Saúde; 2002. v. 26.

2. Brasil. Ministério do Planejamento. Orçamento e Gestão. Síntese de indicadores sociais: 2003. Rio de Janeiro: Instituto Brasileiro de Geografia e Estatística; 2004. [Estudos e pesquisas. Informação Demográfica e Socioeconômica; n. 12].

3. Ribeiro KP. O enfermeiro no serviço de atendimento ao politraumatizado. In: Freire E. Trauma: a doença dos séculos. São Paulo: Atheneu; 2001. v . 1. p. 499-508.

4. Malvestio MAA, Sousa RMC. Suporte avançado à vida: atendimento a vítimas de acidentes de trânsito. Rev Saúde Pública. 2002; 36(5): 584-9.

5. Junqueira Júnior G, Pinto RD, Praetzel R, Pinto RD, Camargo S. Estudo epidemiológico dos atendimentos do SAMU - Serviço de Atendimento Médico de Urgência. Rev HPS. 1997; 43:22-4.

6. Pereira RF, Pinto RD, Ciconet, RM. Caracterização dos atendimentos prestados pelo SAMU. Rev HPS. 1999/2000; 45/46:6-9.

7. Rio Grande do Sul. Departamento de Trânsito. Acidentes de trânsito com vítimas no Rio Grande do Sul: comparativo 1997 a 2003 [monografia na Internet]. Porto Alegre; [2004?]. [citado 2004 Abr 16]. Disponível em: http:// www.detran.rs.gov.br/estatisticas/2003/acid_97_2003.htm.

8. Camargos EF, Peixoto ER, Rocha IS, Amaral MA, Nogueira MARJ, Velloso GR. Acidentes de trânsito em Brasília. Caracterização dos acidentes e das vítimas atendidas no Hospital de Base do Distrito Federal. Rev Saúde Dist Fed. 1997; 8(1):22-6.

9. Karstein AA, Burin JR, Arantes RKC, Falavinha RS. Análise epidemiológica das vítimas atendidas pelo SIATE e transportadas ao Hospital Cajuru. Rev Bras Ortop. 1996; 31(6):485-90.

10. Andrade SM, Mello Jorge MHP. Características das vítimas por acidentes de transporte terrestre em município da Região Sul do Brasil. Rev Saúde Pública. 2000; 34(2):149-56.

11. Rodrigues LA, Miller ELC. Trauma por acidentes de veículos em rodovias. In: Freire E. Trauma: a doença dos séculos. São Paulo: Atheneu; 2001. v.1. cap. 21. p. 349-72.

12. Liberman M, Mulder D, Sampalis J. Advanced or basic life support for trauma: meta-analysis and critical review of the literature. J Trauma. 2000; 49(4):584-99.

13. Thomaz RR, Lima FV. Atuação do enfermeiro no atendimento pré-hospitalar na cidade de São Paulo. Acta Paul Enfermagem. 2000; 13(3):59-65. 